\title{
Estimating the Local Burden of Disease During the First Wave of the COVID-19 Epidemic in England, Using Different Data Sources from Changing Surveillance Practices
}

\author{
Emily S Nightingale ( $\nabla$ emily.nightingale@lshtm.ac.uk) \\ London School of Hygiene \& Tropical Medicine \\ Sam Abbott \\ London School of Hygiene \& Tropical Medicine \\ Timothy W Russell \\ London School of Hygiene \& Tropical Medicine

\section{Rachel Lowe} \\ London School of Hygiene \& Tropical Medicine \\ Graham F Medley \\ London School of Hygiene \& Tropical Medicine \\ Oliver J Brady \\ London School of Hygiene \& Tropical Medicine
}

\section{Research Article}

Keywords: COVID-19, infections

Posted Date: December 16th, 2021

DOI: https://doi.org/10.21203/rs.3.rs-1113627/v1

License: (c) This work is licensed under a Creative Commons Attribution 4.0 International License. Read Full License

Version of Record: A version of this preprint was published at BMC Public Health on April 11th, 2022. See the published version at https://doi.org/10.1186/s12889-022-13069-0. 


\section{Abstract}

Background

The COVID-19 epidemic has differentially impacted communities across England, with regional variation in rates of confirmed cases, hospitalisations and deaths. Measurement of this burden changed substantially over the first months, as surveillance was expanded to accommodate the escalating epidemic. Laboratory confirmation was initially restricted to clinical need ("pillar 1") before expanding to community-wide symptomatics ("pillar 2"). This study aimed to ascertain whether inconsistent measurement of case data resulting from varying testing coverage could be reconciled by drawing inference from COVID-19related deaths.

Methods

We fit a Bayesian spatio-temporal model to weekly COVID-19-related deaths per local authority (LTLA) throughout the first wave (1 January - 30 June 2020 ), adjusting for the local epidemic timing and the age, deprivation and ethnic composition of its population. We combined predictions from this model with case data under community-wide, symptomatic testing and infection prevalence estimates from the ONS infection survey, to infer the likely trajectory of infections implied by the deaths in each LTLA.

Results

A model including temporally- and spatially-correlated random effects was found to best accommodate the observed variation in COVID-19-related deaths, after accounting for local population characteristics. Predicted case counts under community-wide symptomatic testing suggest a total of $275,000-420,000$ cases over the first wave - a median of over 100,000 additional to the total confirmed in practice under varying testing coverage. This translates to a peak incidence of around 200,000 total infections per week across England. The extent to which estimated total infections are reflected in confirmed case counts was found to vary substantially across LTLAs, ranging from $7 \%$ in Leicester to $96 \%$ in Gloucester with a median of $23 \%$.

Conclusions

Limitations in testing capacity biased the observed trajectory of COVID-19 infections throughout the first wave. Basing inference on COVID-19-related mortality and higher-coverage testing later in the time period, we could explore the extent of this bias more explicitly. Evidence points towards substantial underrepresentation of initial growth and peak magnitude of infections nationally, to which different parts of the country contribute unequally.

\section{Introduction}

The COVID-19 epidemic has impacted communities heterogeneously across England since evidence first emerged of local transmission in March 2020 (1). Spatio-temporal patterns in transmission - driven, for example, by connectivity between regions, timing of initial exposure and impact of control measures offer valuable insight into the development of the epidemic. However, such patterns are difficult to observe and interpret from raw reported case data alone, due to uneven vulnerabilities in the local population and changes in surveillance policy over time (2).

In particular, laboratory confirmation of cases was initially restricted to urgent clinical need of patients and healthcare staff ("pillar 1") before being expanded to encompass all symptomatic cases in the wider community ("pillar 2") from the 18th May 2020 (3). These data therefore reflect different subsets of total infections at different points of the epidemic. Deaths - in particular the broad class of COVID-19-related deaths, including both test-confirmed and clinically suspected cases (where the disease is considered to be the primary cause of death or a contributing factor) - can be considered more consistently recorded over time. We seek to exploit the biological link between the two sources of data to obtain a clearer picture of the burden of COVID-19 during the first wave, and to quantify the extent of under-ascertainment - by which we mean the gap between reported, confirmed cases and total infections - during scale up of testing.

Observed variation in the rate of COVID-19-related deaths can be considered a result of two spatially varying components: variation in incidence of infection and variation in fatality risk among those infected. Several individual-level characteristics have been highlighted as risk factors for COVID-19 case fatality including age, deprivation and belonging to certain ethnic groups - all of which are themselves geographically clustered (Figure S1). The influence of this on a population level is evident in local summaries of mortality rates in England and Wales (4), and will lead to substantial variation in the number of infections which give rise to observed deaths among the populations of different local areas. These factors should therefore be taken into account in order to understand how the relative number of deaths to infections varies over space and time. Changes in surveillance affect the probability of an infection being reported as a case and are therefore also important to account for when observing changes in the relative number of deaths to cases over time.

Previous studies have demonstrated several different methods for estimating the number of cases from reported deaths. Jombart et al. (5) offered an early attempt to infer symptomatic cases from the occurrence of a single death, concluding that there would have been in the region of several hundreds of cases by the time the first death was recorded. Russell et al. (6) proposed an approach based on published estimates of baseline case-fatality rates to estimate the proportion of unreported cases over time directly, at national and regional levels for a range of early-affected countries. Nicholson et al. (7) further discuss the impact of ascertainment bias in the UK's surveillance systems and present an approach to quantify it through a joint analysis of targeted symptomatic and randomised testing data.

Other work exploring variation in mortality between local geographies of the UK has not accounted for the lack of independence between the units of interest, implicitly assuming that geographical regions can be considered independent after adjusting for a set of population covariates (8). However, small and frequently zero counts in death data at a local level can limit precision of estimates when analysed independently. Sartorius et al. (9) do explicitly account for 
this dependence, adding a data-driven spatial structure in the form of correlated random effects within a mechanistic SEIR model, but fit to pillar one case counts only, assuming these represent a fitted proportion of total infections constrained between $5-40 \%$, as informed by two systematic reviews of the asymptomatic proportion. This does not account for the proportion of individuals who are symptomatic but do not obtain a confirmed diagnosis.

This analysis aims to extend the concept of inferring infections and cases from deaths down to a local level while accounting for varying population characteristics, timing of first exposure and other unexplained sources of spatial correlation. With this approach we pursue a clearer understanding of the relative burden of disease across the country and how each locality contributes to the national picture.

\section{Materials And Methods}

Data sources

Anonymised linelists of reported COVID-19-related deaths between the 1st of January and 30th of June 2020 were provided by Public Health England (PHE). COVID-19-related deaths were considered to include those with COVID-19 recorded as an underlying cause, or where COVID-19 was mentioned as a contributing factor but not specified as cause of death. These two categories included a total of 52,560 reported deaths in England which occurred between 2020-01-05 and 2020-06-28. Counts were then aggregated by lower-tier local authority (LTLA), week of death (counted from Wednesday 1st January) and 10year age group. Aggregation by week was chosen in order to avoid excessive zero or low counts and potential day-of-week reporting effects, and to obtain a smoother representation of the epidemic curve. Records which did not have a LTLA provided $(n=74)$ were excluded. Local authority shapefiles and single-age population estimates were obtained from the Office for National Statistics $(\mathrm{ONS})(10,11)$ and matched to the aggregated death data.

LTLAs can be classified into one of four geographical categories: London borough (10.3\% of total LTLAs), metropolitan district (11.5\%), non-metropolitan district $(60.3 \%)$ and unitary authority (17.9\%). The former two categories capture the major urban areas of the country (including Birmingham, Liverpool, Manchester, Sheffield, Leeds and Newcastle) with high connectivity both nationally and internationally, while the latter capture predominantly rural areas and smaller towns or cities.

PCR-confirmed pre-P2 and post-P2 cases (i.e. COVID-19 infections identified through both pillar 1 and pillar 2 surveillance) were obtained from the same source and aggregated to the same spatial and temporal resolution. Finally, estimates of infection prevalence in England were obtained from the ONS COVID19 infection survey pilot (12) which was initiated in May 2020. These are presented as an estimated percentage (plus $95 \%$ confidence interval based on the survey sample size) of the population who would test positive via PCR for COVID-19 during rolling fortnightly intervals.

Case definitions

For the remainder of the paper, infections confirmed with a positive PCR test and recorded in official case data prior to the expansion of symptomatic community testing on the 18th of May will be referred to as pre-P2 cases, and infections confirmed following expansion of testing will be referred to as postP2 cases. It is noted that, due to piloting of pillar 2 testing among high-risk groups, a proportion of pre-P2 cases will have been detected via the pillar 2 route. We conservatively define the surveillance policy change from the point at which pillar 2 was fully available to all symptomatic individuals - assuming that case data from this point most accurately reflect the increased coverage of the expanded system - and define the terminology according to this distinction. We will also introduce the concept of predicted-P1+P2 cases, meaning the predicted infections which would have been PCR-confirmed in the hypothetical scenario in which symptomatic community testing had been in place since the beginning of the epidemic (January 2020). These predicted-P1+P2 cases form a subset of total symptomatic cases, conditional on the additional criteria that the case must be both symptomatic and seek and obtain a confirmatory positive test result. Lateral flow devices were not introduced for asymptomatic testing until later in the year (3) and therefore are not considered here. All references to deaths imply COVID-19-related deaths, i.e., those where either PCR-confirmed or clinically diagnosed COVID-19 infection is recorded on the death certificate.

\section{Model Structure}

Bayesian mixed effects models for deaths per week and per LTLA were fitted using integrated nested Laplace approximation (INLA), implemented via the $\mathrm{R}$ package $R$-INLA $(13,14)$.

To facilitate comparison in observed deaths across local authorities with different population age distributions, age-adjusted expected deaths, $E$, were calculated for each LTLA to serve as an offset in models. Expected counts were based on age-specific weekly mortality rates averaged over the observed time period and over the country as a whole. See Supplementary materials for details. Weekly reported deaths in LTLAi were assumed to follow a negative binomial distribution, with log link function, offset by $\log \left(E_{i}\right)$.

In addition to age, two population level characteristics were considered as risk factors for case fatality: level of deprivation and distribution of ethnicity. The Index of Multiple Deprivation (IMD) score is defined as a relative measure of deprivation between Lower Super Output Areas (LSOAs) and incorporates a range of social, economic and health factors (15). IMD scores were aggregated to the median across all LSOAs in each LTLA and categorised by quintiles. To account for the heterogeneous distribution of ethnicity across the country, the percentage of minority ethnic groups in each LTLA population (relative to white as the national majority) was calculated according to estimates from the most recent census (16).

The temporal dependence in the data was modelled using a combination of random effects with random walk (RW) correlation structures (17). A second-order random walk (RW2) on the number of weeks since the first observed death (the "epidemic" week) was intended to capture the shifted epidemic curve in each LA. Additionally, a first-order random walk (RW1) on calendar week was included to capture any overall deviations from these epidemic trends (potentially as a result of policy and behavioural change). As such, the number of deaths in any one LTLA during one week are a priori assumed to be correlated with the 
number of deaths across the prior two weeks. Models in which the second-order RW on epidemic week was fitted separately within each of the four geography categories were also considered.

Models without any specified spatial structure were compared to those with independent and identically-distributed (IID) random effects per LTLA, and with a combination of IID and structured, conditional auto-regressive effects (as described by Besag, York and Mollié (18), hereafter referred to as BYM), parameterised with a mixing parameter $\phi$ between the two (19). The latter allowed assessment of the contribution of local spatial correlation to the fit of the model, relative to purely random (IID) variation.

Six models were fitted and compared:

(A) Baseline Observed deaths log(E) (offset) + Overall epidemic trend (RW2) + calendar week trend (RW1) + covariates (IMD, \% minority); no spatial structure.

(B) A + geography-dependent epidemic trends

(C) A + IID spatial structure

(D) B + IID spatial structure

(E) A + BYM spatial structure

(F) B + BYM spatial structure

The distributions of structured random effects (spatial and temporal) were fit with penalised complexity priors on the precision and BYM mixing parameters (20), and fixed effects fit with weakly-informative gaussian priors centred at zero. A more detailed specification of all models can be found in the supplementary materials.

All analyses were performed in R version 3.6 .3 (2020-02-29). All code used to run these analyses have been made available at https://doi.org/10.5281/zenodo.5763664.

Model Comparison

Models were compared using WAIC (21) and log score (22). Pearson residuals between fitted values and observed were averaged per LTLA and mapped as a visualisation of the spatial structure unexplained by each model. Posterior samples $(n=1,000)$ were drawn to explore the uncertainty in predictions and aggregated over LTLAs to give total trajectories over time.

Comparison to post-P2 cases

It was assumed that post-P2 cases (swabbed from 18th May onwards) were reflective of the higher coverage surveillance and less obscured by capacity constraints. A fixed lag of one week between date of swabbing and date of death was applied to infer predicted-P1+P2 cases from modelled deaths in the primary analysis, while a sensitivity analysis was conducted assuming two- and three-week lags. This choice was informed by the swab-death delay distribution observed in this dataset (median 6 days, IQR 8 days), while also considering an external report from the COVID Clinical Information Network (COCIN) (23) which suggested an overall longer and more varied distribution (median 13 days, IQR 14 days) between onset of symptoms and death. The possibility was considered that the lag between testing and death may have been shorter early in the epidemic, with cases predominantly being tested in a hospital setting when symptoms were already severe. However, the available data on swabbing and death dates did not suggest a difference between pre- and post-P2 cases (median 6 days pre-P2 and 7 days post-P2, with equal quartiles of 3-11 days), and therefore one fixed lag was assumed for the entire period. It was assumed that variation over this period of time in the ratio of post-P2 cases to deaths would be predominantly a result of varying completeness of observation of cases, rather than of a difference in underlying case-fatality risk.

The approach taken to infer predicted-P1+P2 cases from reported deaths consisted of three steps. First, smoothed trajectories of deaths per week and per LTLA, corrected for spatial heterogeneity in case-fatality risk factors, were obtained from the fitted model $(1,000$ posterior samples predicted at averaged covariate values with non-age-stratified population offset). An LTLA-level ratio of cases per covid-related death (post-P2 case per death ratio, CPDR) was then estimated for every week beyond the 18th May and for each posterior sample, lagging the modelled death counts by one week (two and three weeks for the sensitivity analysis) and comparing to post-P2 cases. CPDRs were summarised over all post-P2 weeks to obtain a median and IQR for each LTLA, which were then used to scale up the posterior samples over the whole time period. This yielded an estimate of the magnitude of cases giving rise to those deaths, which would have been detected under expanded surveillance. 
Inferring infection and rate of detection

The previous steps yield estimated local trajectories of COVID-19 infections which would have been detected through combined hospital and communitybased symptomatic testing, had such capacity been available throughout the first epidemic wave. However, post-P2 cases detected under expanded surveillance remain a subset of the total number of infections. The ONS COVID-19 infection survey pilot (12) suggested that per fortnight between the 27th April and 24 th May around $0.25 \%$ of the population of England would have tested positive for COVID-19, with this percentage steadily decreasing to $0.03 \%$ by the beginning of July. To investigate the gap between total infection incidence and detected cases, these data were combined with post-P2 case counts over the same period to infer a rate of detection under expanded surveillance (see Supplementary Materials D). This rate of detection was then applied to the entire trajectories of predicted-P1+P2 cases to estimate the number of infections represented by those detected cases. Observed pre- and post-P2 counts could then be compared to these estimated infections to infer the percentage of infections detected over time and within each LTLA.

\section{Results}

A summary of the observed incidence of covid-related deaths and pre-/post-P2 confirmed cases is shown in Figure 1. Over time, the early exposure of London is clear in both deaths and confirmed cases, with the two epidemic curves following a similar shape and peaking prior to the other geographies. Outside of London, confirmed case counts appear to be truncated between the end of March and the end of April, approximately coinciding with the implementation of the national lockdown on the 23rd of March.

Overall, COVID-19-related mortality rates ranged from 10 per 100,000 in South Hampshire to 196 per 100,000 in Hertsmere (median [IQR]: 90.6 [71.4, 112.1]). Cumulative incidence of confirmed cases was more varied between LTLAs, ranging from 71 per 100,000 in Torridge in North Devon, to 1040 per 100,000 in the East Midlands city of Leicester (median [IQR]: 379.2 [298.3, 491.5]). Supplementary figure S1 illustrates the substantial variation in the population characteristics assumed to contribute to case-fatality risk across the country.

Model selection

By comparison of information criteria (WAIC) and cross-validated log score, it is clear that adjustment for epidemic timing and the specified fatality risk covariates (model A) were insufficient alone to explain the spatial distribution of deaths across England. Out of the six candidate models, the BYM spatial model and temporal trends specific to the geography of the LTLA was selected as offering the lowest WAIC and best cross-validated fit (model F) (Table 1). Models with unstructured, IID random effects per LTLA performed comparably to the BYM model and the overall magnitude of error appeared to be reduced, but spatial structure in the residuals was still evident (Supplementary figure S2).

Table 1

Overall model comparison by WAIC and log score

\begin{tabular}{|lllll|}
\hline Model & WAIC & Log score & Diff WAIC & Diff log score \\
\hline B + BYM spatial & 24750.37 & 2.601 & - & - \\
\hline B + IID spatial & 24800.96 & 2.606 & 50.59 & 0.005 \\
\hline A + BYM spatial & 25601.8 & 2.69 & 851.431 & 0.089 \\
\hline A + IID spatial & 25664.73 & 2.697 & 914.357 & 0.096 \\
\hline Geog-specific temporal (B) & 26343.81 & 2.768 & 1593.441 & 0.167 \\
\hline Temporal only (A) & 26865.43 & 2.823 & 2115.061 & 0.222 \\
\hline
\end{tabular}

Final model

The final model suggested strong associations between weekly rates of COVID-19-related deaths in a LTLA, quintiles of deprivation score and proportion of minority ethnicities in the population ( $R R=1.27$ with 95\% Credible Interval $\mathrm{Crl}$ [1.10 - 1.47] between the 1st and 4th quintiles of IMD; RR = 1.01 [1.006 - 1.015] per percentage increase in minority ethnic population), after adjusting for the size and age distribution of the local population (Supplementary table S2). Despite a clear monotonic trend through the first four quintiles of deprivation score, the difference between the 1st and 5th (most deprived) quintiles dropped slightly and was estimated with a wider $\mathrm{Crl}(\mathrm{RR}=1.21[0.97,1.49])$, perhaps due to the smaller number of LTLAs which fall into this category. Differences in the shape of the epidemic between each geography type were best captured by four separately fitted trends as opposed to one overall trend, and residual heterogeneity between LTLAs (i.e., not captured by covariates) was explained by a combination of spatial correlation and random noise.

Posterior samples drawn from the selected model illustrated a close fit to the epidemic trajectories overall and within each specific geography (Figure 2). Fits for a random sample of individual LTLAs are illustrated in Supplementary figure S3.

The fitted posterior for the BYM mixing parameter, $\Phi$, implies that at least $86 \%$ (posterior mean 95\%, 95\% Crl: [86 - $99 \%$ ]) of the residual spatial variation (accounting for the specified covariates and temporal trends) could be explained by correlation between neighbouring LTLAs as opposed to random noise. This suggests that there is correlation in observed mortality in neighbouring areas which is not explained by similarities in the size, age distribution, ethnic composition or deprivation level of their populations. A decomposition of the fitted spatial random effects for each LTLA is illustrated in Supplementary figure S4.

Comparison to post-P2 cases

Page 5/14 
Prior to the expansion of pillar 2 surveillance, the median CPDR per LTLA was 4.1 confirmed cases per covid-related death (IQR [3.4,5.0]). From the 18th of May onwards, this increased to a median of 5.2 with more variation between LTLAs (IQR $[3.3,8.6])$. Further detail of the spatial heterogeneity in CPDR across the country is illustrated in Supplementary figure S5.

Figure 3: Predicted P1+P2 cases, according to lagged and scaled-up predictions from the selected model for COVID-19-related deaths, in total (left) and aggregated by geography type (right). Intervals shown are 50-98\% quantiles of the predicted distribution. Observed totals of confirmed cases per week are indicated by black points - unfilled prior to P2-expansion and filled post-P2 expansion. Predicted-P1+P2 cases suggest the potential shape and magnitude of the first wave peak if community symptomatic testing (pillar 2) - in addition to hospital-based testing (pillar 1) - had been available from the beginning of the epidemic.

Table 2

Summary of observed and predicted-P1+P2 case counts over the first wave, nationally and by geography.

\begin{tabular}{|llll|}
\hline & $\begin{array}{l}\text { Observed, test- confirmed cases } \\
\text { (up to week starting 2020-06-17) }\end{array}$ & $\begin{array}{l}\text { Predicted } \\
\text { (median [98\% CrI) }\end{array}$ & $\begin{array}{l}\text { Percentage } \\
\text { difference }\end{array}$ \\
\hline England total & 231,817 & $335,083[275,482-418,847]$ & $44.5[18.8-80.7]$ \\
\hline London Borough & 33,399 & $43,664[35,881-51,337]$ & $30.7[7.4-53.7]$ \\
\hline Metropolitan District & 64,007 & $109,717[95,734-129,216]$ & $71.4[49.6-101.9]$ \\
\hline Non-metropolitan District & 79,441 & $97,786[78,764-122,095]$ & $23.1[-0.9-53.7]$ \\
\hline Unitary Authority & 54,970 & $83,723[67,673-110,968]$ & $52.3[23.1-101.9]$ \\
\hline
\end{tabular}

The four geography types contribute unevenly to this difference. In London, the reconstructed counts suggest a relative under-representation of the peak incidence in observed confirmed cases, which narrows relatively rapidly from April onwards as numbers decline and testing capacity increases. The implied under-ascertainment in London is of a much smaller magnitude than the other three geographies; in particular for metropolitan districts and unitary authorities, results suggest that, at the height of the epidemic, confirmed cases potentially constituted less than half of the symptomatic cases which would have been detected under the expanded system. For the predominantly rural non-metropolitan districts the difference at the peak is less substantial, though still greater than that of London. From late-April, total confirmed case incidence across these LTLAs actually exceeds the reconstructed counts, by a small margin which diminishes towards the beginning of the summer.

Assuming a longer two-week lag between testing and death yields a much larger difference of $86 \%$, and for three weeks this increases further to $135 \%$. A comparison of reconstructed national totals based on three different lags is included in Supplementary Table S3 and illustrated in Figure 4. It is clearly shown that assuming a longer lag between case confirmation and death yields a higher and earlier peak in the reconstructed trajectory of cases.

Estimation of total infections

Figure 5 illustrates the estimated national incidence of infection according to the ONS infection survey pilot, alongside total observed and reconstructed cases across the country. Comparison of observed cases from weeks starting 18th May to 15th June with these estimated total infections suggested an overall rate of detection of $25 \%$ ( $95 \% \mathrm{Cl}$ propagated from infection prevalence estimates: $13-58 \%)$. The total wave of infections over the entire period implied by this rate of detection is indicated by the grey curve.

This yields a cumulative total of 1.3 million infections ( $98 \% \mathrm{Crl} 1.04$ to 1.74 million) throughout the first wave, of which the observed confirmed cases ( $\mathrm{n}=$ $231,817)$ constitute $17.5 \%$ (98\% Crl 13.3-22.3\%).

Within each LTLA, cumulative incidence of confirmed cases constituted a median of $23 \%$ of estimate total infections (Figure $6 \mathrm{~A}$ ). The highest rates of detection were found in Gloucester and Teignbridge in the south-west, both with estimates of over $96 \%$ (98\% Crls [87\%, 110\%] and [81\%, 121\%], respectively), while less than $7 \%$ detection was estimated in Leicester, Tunbridge Wells and Bradford ( $98 \%$ Crls [3\%, 11\%], [6\%, 15\%] and [4\%, 10\%], respectively). See Supplementary Figure S6 for predicted trajectories in these LTLAs. Figure 6B presents the estimated detection rate for each LTLA compared to the total observed incidence, grouped by region. In most regions, greater observed incidence coincides with poorer detection of total infections, perhaps reflecting the impact of reaching testing capacity. However, in London and the North, the trend leans more into the opposite direction. By week, the level of underascertainment decreases in magnitude from February to April and settles between 25 and $30 \%$ from late-April to June (Figure $6 \mathrm{C}$ ).

The final predicted-P1+P2 and total infections for the entire time series in each LTLA are included in Supplementary materials E.

\section{Discussion}

This analysis has demonstrated that it is possible to generate plausible case burden estimates from COVID-19-related death data and, in doing so, investigate the impact of changes in surveillance practices over the first months of the epidemic.

The model development process highlights a clear spatial structure to the incidence of covid-related deaths at a sub-national level, which is not explained by variation in the timing of initial exposure (epidemic week), or well-documented risk factors of COVID-19 death (age, deprivation and ethnic distribution in the local population). Similarities in risk between neighbouring LTLAs can be an important factor to consider for the design of local mitigation strategies, particularly in response to the detection of new variants. 
Assuming that the epidemic curve of deaths represents a specific subset of total symptomatic - i.e., detectable under Pillar 1 and 2 testing strategies infections, this analysis suggests that over 100,000 additional cases may have been counted across the country in the absence of the initial constraints on testing capacity. The uncertainty around this estimate is relatively broad (98\% $\mathrm{Crl}[44,000-250,000])$, predominantly as a result of uncertainty in the case-perdeath ratio used to translate between the two measures. The increased heterogeneity between LTLAs in estimated CPDR following pillar 2 expansion may to some extent be attributed to much lower counts of both cases and deaths as the epidemic waned, and the occurrence of local outbreaks. Overall, we estimate around four post-P2 confirmed cases per covid-related death across all LTLAs, or equivalently a rate of 0.25 deaths per case. This is higher than estimates of the case-fatality rate i.e. the rate of deaths among confirmed cases .

since we instead due to our broader definition of covid-related deaths and .

Cases ascertained, even under the expanded system, remain a subset of total infections (sometimes referred to as symptomatic and asymptomatic cases). The symptomatic case estimates obtained here were therefore combined with estimates of infection incidence from the ONS's pilot survey in order to explore the rate of detection over time and between LTLAs. This investigation suggested that, following the roll-out of symptomatic community testing, around a quarter of infections in England were being detected - a value consistent with estimates obtained by Colman et al. (24) for the period of June to November 2020 - compared to only around $10 \%$ during the first months of the pandemic.

The extent of this under-ascertainment was found to vary not only over time alongside the expansion of testing capacity, but also between LTLAs. Comparing the final model fit to the observed deaths suggested relatively little deviation of each LTLA from the fitted geography-specific trends, yet the reconstructed infections differ from observed test positives with much more variation between LTLAs. This demonstrates the sporadic nature of case observation over time and space and highlights certain LTLAs in which surveillance was perhaps more strongly impacted by testing constraints. These differences may in part be attributed to local variation in the relationship between cases and deaths which isn't sufficiently captured by the assumed case-fatality covariates. We therefore advise that LTLA-specific estimates should be interpreted with consideration of the local context.

Early projections based on critical care admissions by Jit et al. (25) suggested an incidence of over 8,000 infections per day in the UK by mid-March 2020. Assuming detection of $25 \%$ of infections under hospital and community symptomatic testing, from this study we estimate a total of around 111,000 infections during the two middle weeks of March, equating to an average of just under 8,000 per day in England alone. On the other hand, via a mechanistic modelling approach, another study estimated daily total infections in the UK to have reached in the region of several hundred thousand by late-March (26). Genomic analysis suggests that importations into the UK alone peaked mid-March with up to 1,000 per day (27).

Russell et al. (6) took a data-driven approach in estimating that the peak incidence of symptomatic infections across the UK had occurred by mid-April 2020 with a magnitude of around 100,000 per day, and concluded that during March only $3-10 \%$ of such cases were being detected. This suggests a substantially higher peak than our estimate for England alone of just over 200,000 tota/ infections per week - on average 28,000 per day - even accounting for the distribution of population between the constituent countries. Our estimates suggest that the percentage of these total infections reflected in confirmed case counts varied from around $7 \%$ at the start of March to $11 \%$ at the end, slightly higher than the detection rate Russell et al. estimated among symptomatic infections. Overall, estimates of infection incidence appear to be variable across studies, at least in part due differences in case definitions and aggregation over space and time.

Limitations

The interpretation of these findings depends on several key assumptions, most importantly that variability over time in the ratio of confirmed cases to COVID19-related deaths is predominantly the result of varying accessibility of testing. It is however plausible that fatality risk would have varied over time, potentially increasing towards the peak of the wave due to strain on hospitals forcing re-prioritisation of care or decreasing later on as treatment options improved. Also, it was assumed that variability in the delay from swabbing to death on the individual level would be diluted by aggregation, hence a fixed-value lag (with its influence explored in sensitivity analysis) would suffice. Summarising observed delays between swabbing and death within the available data gave no reason to suggest a difference between the time periods pre- and post-pillar 2 expansion, therefore the same fixed lag was assumed throughout the epidemic wave. A more exact approach, however, would have been to incorporate the full distribution of swab-death delays and redistribute the observed deaths in time according to an imputed point of detection.

Only two broad characteristics were considered as case-fatality risk factors, which essentially serve as proxy measures for complex combinations of underlying comorbidities and health indicators across the population. Dichotomising ethnicity in a population into "majority" and "minority" groups is crude, given that risk has been found to differ between ethnic groups in different ways (28). In individual level analysis, comorbidities such as cardiovascular disease, diabetes, and cancer were shown to have an association with mortality after adjusting for both ethnicity and deprivation level (29). The local prevalence of such conditions is however a component in the calculation of the deprivation score used here. There are likely nuances and complex interactions between granular risk factors for mortality $(30,31)$ which are not yet understood in sufficient detail to be explicitly defined in such a model. The measures used here are therefore intended to capture high-level, population-level differences.

Finally, this approach does not account for the dynamics of transmission in and around long-term residential care facilities during the early months of the pandemic, within which many deaths during the first wave occurred (32). The nature of infections in these settings - with respect to mortality, testing and management - is different to that which occurred in the wider community, yet both care home and community deaths were treated equally in this analysis. For LTLAs with a particularly large care home population, the estimated case-per-death ratio may be higher than it would have been excluding these particularly vulnerable individuals. However, adjusting for the age distribution of the LTLA population in the underlying deaths model should at least in part attenuate this source of variation. This study aimed to explore broad, population-level patterns in incidence of deaths and detection of cases, whereas characterising the 
contribution of incidence within residential care settings would require a more fine-scaled, context-specific analysis. There was further substantial transmission within healthcare settings which we have not included separately (33).

\section{Conclusions}

Effective and efficient control of an infectious disease epidemic relies on appropriate quantification of risk at a local level from available surveillance data. However, there are many reasons for which such data may not be equally representative of disease burden across different regions and populations. In the case of the COVID-19 epidemic in England, it is known that limitations in testing capacity distorted the observed trajectory of cases during the first wave. In this analysis, by combining more consistently reported data on deaths and more representative case data from later in the epidemic, it was possible to reconstruct a plausible trajectory of symptomatic cases which could have been detected in the absence of the early testing constraints, and further to infer the total number of infections these reported cases would represent. This facilitated a comparison between the two testing policies and highlighted heterogeneity in case ascertainment across different regions of the country.

The burden of disease and impact of the response to this pandemic will be evaluated in detail for years to come. Considering how changes in surveillance policy can obscure the spread of an epidemic - using methods such as those demonstrated here - will be essential, in particular for understanding the consequences of the country's initial level of pandemic preparedness.

\section{Abbreviations}

\begin{tabular}{|ll|}
\hline BYM & Besag York Mollié \\
\hline CPDR & Cases Per Death Ratio \\
\hline CO-CIN & COVID Clinical Information Network \\
\hline CrI & Credible Interval \\
\hline IID & Independent and Identically Distributed \\
\hline IMD & Index of Multiple Deprivation \\
\hline INLA & Integrated Nested Laplace Approximation \\
\hline IQR & Inter-Quartile Range \\
\hline LSOA & Lower Super Output Area \\
\hline LTLA & Lower Tier Local Authority \\
\hline ONS & Office for National Statistics \\
\hline PCR & Polymerase chain reaction \\
\hline RW & Random Walk \\
\hline WAIC & Widely Applicable Information Criterion \\
\hline
\end{tabular}

\section{Declarations}

\section{Author contributions}

ESN, GFM and OJB conceptualised the analysis and GFM and OJB supervised the work. ESN performed the analyses and prepared all figures. SA, TWR and $\mathrm{RL}$ gave feedback on methodology and presentation of results. ESN wrote the manuscript. SA, RL, GFM and OJB reviewed and edited the manuscript. ESN produced the remote code repository. All co-authors provided feedback on the analyses and the manuscript, and agreed with the final submitted version. Authors who were part of the Centre for Mathematical Modelling of Infectious Disease COVID-19 working group each contributed in processing, cleaning and interpretation of data, interpreted findings, contributed to the manuscript, and approved the work for publication.

\section{Funding}

ESN and GFM are funded by the Bill and Melinda Gates Foundation (ESN: OPP1183986, GFM: OPP1184344). OJB and SA are funded by the Wellcome Trust (OJB: 206471/Z/17/Z, SA: 210758/Z/18/Z, TWR: 206250/Z/17/Z). RL is funded by the Royal Society (Dorothy Hodgkin Fellowship).

The author(s) declare(s) that they have no competing interests.

\section{Ethical statement}

Approval for the use of anonymised linelist data was granted by Public Health England and the Department for Health and Social Care. Consent of individuals was not required as no patient identifiable information was used. 


\section{Availability of data and materials}

\section{The datasets supporting the conclusions of this article are available at https://doi.org/10.5281/zenodo.5763664.}

All methods were performed in accordance with the relevant guidelines and regulations regarding analysis of human data, including SAMPL guidelines for reporting of statistical analyses.

\section{Consent to publish}

Consent to publish was not required as this manuscript contains no individually identifiable details or images.

\section{Acknowledgments}

The authors would like to thank Christopher Jewel and Jonathon Reed for valuable discussion during the early stages of this work.

The following authors were part of the Centre for Mathematical Modelling of Infectious Disease COVID-19 working group: Eleanor M. Rees, Rosalind M. Eggo, Matthew Quaife, Fiona Yueqian Sun, Carl A. B. Pearson, Kiesha Prem, James D. Munday, Sophie R. Meakin, Graham Medley, Kevin van Zandvoort, W. John Edmunds, Alicia Rosello, Sebastian Funk, Kathleen O’Reilly, Billy J. Quilty, Simon R. Procter, Amy Gimma, Adam J. Kucharski, Arminder K. Deol, Jon C. Emery, Nikos I. Bosse, Hamish P. Gibbs, David Simons, Stéphane Hué, Christopher I. Jarvis, Petra Klepac, Yang Liu, Anna M. Foss, Charlie Diamond, C. Julian Villabona-Arenas, Akira Endo, Rein M. G. J. Houben, Stefan Flasche, Timothy W. Russell, Samuel Clifford, Gwenan M. Knight, Joel Hellewell, Nicholas G. Davies, Katherine E. Atkins, Damien C. Tully, Megan Auzenbergs, Mark Jit \& Rachel Lowe.

The following funding sources are acknowledged as providing funding for the CMMID Covid-19 working group:

This research was partly funded by the Bill \& Melinda Gates Foundation (OPP1183986: ESN). This research was partly funded by the Global Challenges Research Fund (GCRF) project 'RECAP' managed through RCUK and ESRC (ES/P010873/1: TJ). This research was partly funded by the National Institute for Health Research (NIHR) using UK aid from the UK Government to support global health research. The views expressed in this publication are those of the author(s) and not necessarily those of the NIHR or the UK Department of Health and Social Care (Health Protection Research Unit for Modelling Methodology HPRU-2012-10096: TJ). UK MRC (LID DTP MR/N013638/1: QJL; MC_PC_19065: TJ). Authors of this research receive funding from UK Public Health Rapid Support Team funded by the United Kingdom Department of Health and Social Care (TJ). Wellcome Trust (210758/Z/18/Z: SA). The following funding sources are acknowledged as providing funding for the working group authors. Alan Turing Institute (AE). BBSRC LIDP (BB/M009513/1: DS). This research was partly funded by the Bill \& Melinda Gates Foundation (INV-003174: KP, MJ, YL; NTD Modelling Consortium OPP1184344: CABP, GM; OPP1180644: SRP; OPP1191821: KO'R, MA). DFID/Wellcome Trust (Epidemic Preparedness Coronavirus research programme 221303/Z/20/Z: CABP, KvZ). Elrha R2HC/UK DFID/Wellcome Trust/This research was partly funded by the National Institute for Health Research (NIHR) using UK aid from the UK Government to support global health research. The views expressed in this publication are those of the author(s) and not necessarily those of the NIHR or the UK Department of Health and Social Care (KvZ). ERC Starting Grant (\#757688: CJVA, KEA; \#757699: JCE, MQ, RMGJH). This project has received funding from the European Union's Horizon 2020 research and innovation programme-project EpiPose (101003688: KP, MJ, PK, WJE, YL). This research was partly funded by the Global Challenges Research Fund (GCRF) project 'RECAP' managed through RCUK and ESRC (ES/P010873/1: AG, CIJ). HDR UK (MR/S003975/1: RME). Nakajima Foundation (AE). NIHR (16/137/109: BJQ, CD, FYS, MJ, YL; Health Protection Research Unit for Modelling Methodology HPRU-2012-10096: NGD; PR-OD-101720002: AR). Royal Society (Dorothy Hodgkin Fellowship: RL; RP\EAI180004: PK). UK DHSC/UK Aid/NIHR (ITCRZ 03010: HPG). UK MRC (LID DTP MR/N013638/1: EMR; MC_PC 19065: RME; MR/P014658/1: GMK). Wellcome Trust (206250/Z/17/Z: AJK, TWR; 208812/Z/17/Z: SC, SFlasche; 210758/Z/18/Z: JDM, JH, NIB, SFunk, SRM). No funding (AKD, AMF, DCT, SH).

\section{References}

1. Holden B, Quinney A, Padfield S, Morton W, Coles S, Manley P, et al. COVID-19: public health management of the first two confirmed cases identified in the UK. Epidemiol Infect [Internet]. 2020 Aug 28 [cited 2020 Oct 15];148. Available from: https://www.ncbi.nlm.nih.gov/pmc/articles/PMC7484301/

2. Sherratt K, Abbott S, Meakin SR, Hellewell J, Munday JD, Bosse N, et al. Exploring surveillance data biases when estimating the reproduction number: with insights into subpopulation transmission of Covid-19 in England. medRxiv. 2021 Mar 18;2020.10.18.20214585.

3. Coronavirus (COVID-19): scaling up testing programmes [Internet]. GOV.UK. [cited 2021 May 14]. Available from: https://www.gov.uk/government/publications/coronavirus-covid-19-scaling-up-testing-programmes

4. Deaths involving COVID-19 by local area and socioeconomic deprivation - Office for National Statistics [Internet]. [cited 2021 Mar 17]. Available from: https://www.ons.gov.uk/peoplepopulationandcommunity/birthsdeathsandmarriages/deaths/bulletins/deathsinvolvingcovid19bylocalareasanddeprivatio

5. Jombart T, van Zandvoort K, Russell TW, Jarvis Cl, Gimma A, Abbott S, et al. Inferring the number of COVID-19 cases from recently reported deaths. Wellcome Open Res. 2020 Apr 27;5:78.

6. Russell TW, Golding N, Hellewell J, Abbott S, Wright L, Pearson CAB, et al. Reconstructing the early global dynamics of under-ascertained COVID-19 cases and infections. BMC Med. 2020 Oct 22;18(1):332. 
7. Nicholson G, Lehmann B, Padellini T, Pouwels KB, Jersakova R, Lomax J, et al. Local prevalence of transmissible SARS-CoV-2 infection: an integrative causal model for debiasing fine-scale targeted testing data [Internet]. 2021 May [cited 2021 Oct 20] p. 2021.05.17.21256818. Available from:

https://www.medrxiv.org/content/10.1101/2021.05.17.21256818v1

8. Verhagen MD, Brazel DM, Dowd JB, Kashnitsky I, Mills MC. Forecasting spatial, socioeconomic and demographic variation in COVID-19 health care demand in England and Wales. BMC Med. 2020 Jun 29;18(1):203.

9. Sartorius B, Lawson AB, Pullan RL. Modelling and predicting the spatio-temporal spread of COVID-19, associated deaths and impact of key risk factors in England. Sci Rep. 2021 Mar 8;11(1):5378.

10. Local Authority Districts (April 2019) Names and Codes in the United Kingdom [Internet]. [cited 2021 Mar 17]. Available from: https://geoportal.statistics.gov.uk/datasets/c3ddcd23a15c4d7985d8b36f1344b1db_0

11. Population estimates for the UK, England and Wales, Scotland and Northern Ireland - Office for National Statistics [Internet]. [cited 2021 Mar 17]. Available from:

https://www.ons.gov.uk/peoplepopulationandcommunity/populationandmigration/populationestimates/bulletins/annualmidyearpopulationestimates/m

12. Coronavirus (COVID-19) Infection Survey: England - Office for National Statistics [Internet]. [cited 2021 Oct 14]. Available from: https://www.ons.gov.uk/peoplepopulationandcommunity/healthandsocialcare/conditionsanddiseases/datasets/coronaviruscovid19infectionsurveydata

13. Rue H, Martino S, Chopin N. Approximate Bayesian inference for latent Gaussian models by using integrated nested Laplace approximations. J R Stat Soc Ser B Stat Methodol. 2009;71(2):319-92.

14. Martins TG, Simpson D, Lindgren F, Rue H. Bayesian computing with INLA: New features. Comput Stat Data Anal. 2013 Nov 1;67:68-83.

15. English indices of deprivation 2019 [Internet]. GOV.UK. [cited 2021 Feb 1]. Available from: https://www.gov.uk/government/statistics/english-indices-ofdeprivation-2019

16. DC2101EW (Ethnic group by sex by age) - Nomis - Official Labour Market Statistics [Internet]. [cited 2021 Feb 1]. Available from: https://www.nomisweb.co.uk/census/2011/dc2101ew

17. Codling EA, Plank MJ, Benhamou S. Random walk models in biology. J R Soc Interface. 2008 Aug 6;5(25):813-34.

18. Besag J, York J, Mollié A. Bayesian image restoration, with two applications in spatial statistics. Ann Inst Stat Math. 1991 Mar 1;43(1):1-20.

19. An intuitive Bayesian spatial model for disease mapping that accounts for scaling - Andrea Riebler, Sigrunn H Sørbye, Daniel Simpson, Håvard Rue, 2016 [Internet]. [cited 2021 Feb 22]. Available from: https://journals.sagepub.com/doi/abs/10.1177/0962280216660421?journalCode=smma

20. Simpson D, Rue H, Riebler A, Martins TG, Sørbye SH. Penalising Model Component Complexity: A Principled, Practical Approach to Constructing Priors. Stat Sci. 2017 Feb;32(1):1-28.

21. Watanabe S. Asymptotic Equivalence of Bayes Cross Validation and Widely Applicable Information Criterion in Singular Learning Theory. J Mach Learn Res. 2010 Dec 1;11:3571-94.

22. Gneiting T, Aftery AER. Strictly Proper Scoring Rules, Prediction, and Estimation. J Am Stat Assoc. 2007;102(477).

23. CO-CIN: COVID-19 - Time from symptom onset until death in UK hospitalised patients, 7 October 2020 [Internet]. GOV.UK. [cited 2020 Nov 20]. Available from: https://www.gov.uk/government/publications/co-cin-covid-19-time-from-symptom-onset-until-death-in-uk-hospitalised-patients-7-october-2020

24. Case fatality rate vs. Total confirmed COVID-19 deaths [Internet]. Our World in Data. [cited 2021 Nov 24]. Available from: https://ourworldindata.org/grapher/deaths-covid-19-vs-case-fatality-rate

25. COVID-19 confirmed deaths in England (to 31 January 2021): report [Internet]. GOV.UK. [cited 2021 Nov 24]. Available from: https://www.gov.uk/government/publications/covid-19-reported-sars-cov-2-deaths-in-england/covid-19-confirmed-deaths-in-england-report

26. Estimating the proportion of SARS-CoV-2 infections reported through diagnostic testing | medRxiv [Internet]. [cited 2021 Oct 19]. Available from: https://www.medrxiv.org/content/10.1101/2021.02.09.21251411v1.full

27. Jit M, Jombart T, Nightingale ES, Endo A, Abbott S, Group LC for MM of IDC-19 W, et al. Estimating number of cases and spread of coronavirus disease (COVID-19) using critical care admissions, United Kingdom, February to March 2020. Eurosurveillance. 2020 May 7;25(18):2000632.

28. Flaxman S, Mishra S, Gandy A, Unwin HJT, Mellan TA, Coupland H, et al. Estimating the effects of non-pharmaceutical interventions on COVID-19 in Europe. Nature. 2020 Aug;584(7820):257-61.

29. Establishment and lineage dynamics of the SARS-CoV-2 epidemic in the UK [Internet]. [cited 2021 Nov 24]. Available from: https://www.science.org/doi/full/10.1126/science.abf2946

30. Collaborative TO, Mathur R, Rentsch CT, Morton CE, Hulme WJ, Schultze A, et al. Ethnic differences in COVID-19 infection, hospitalisation, and mortality: an OpenSAFELY analysis of 17 million adults in England. medRxiv. 2020 Sep 23;2020.09.22.20198754.

31. Williamson EJ, Walker AJ, Bhaskaran K, Bacon S, Bates C, Morton CE, et al. Factors associated with COVID-19-related death using OpenSAFELY. Nature. 2020 Aug;584(7821):430-6.

32. Lassale C, Gaye B, Hamer M, Gale CR, Batty GD. Ethnic disparities in hospitalisation for COVID-19 in England: The role of socioeconomic factors, mental health, and inflammatory and pro-inflammatory factors in a community-based cohort study. Brain Behav Immun. 2020 Aug 1;88:44-9.

33. Townsend MJ, Kyle TK, Stanford FC. Outcomes of COVID-19: disparities in obesity and by ethnicity/race. Int J Obes. 2020 Sep;44(9):1807-9.

34. Gordon AL, Goodman C, Achterberg W, Barker RO, Burns E, Hanratty B, et al. Commentary: COVID in care homes-challenges and dilemmas in healthcare delivery. Age Ageing. 2020 Aug 24;49(5):701-5.

35. Knight GM, Pham TM, Stimson J, Funk S, Jafari Y, Pople D, et al. The contribution of hospital-acquired infections to the COVID-19 epidemic in England in the first half of 2020 [Internet]. 2021 Sep [cited 2021 Nov 24] p. 2021.09.02.21262480. Available from:

Page $10 / 14$ 


\section{Figures}
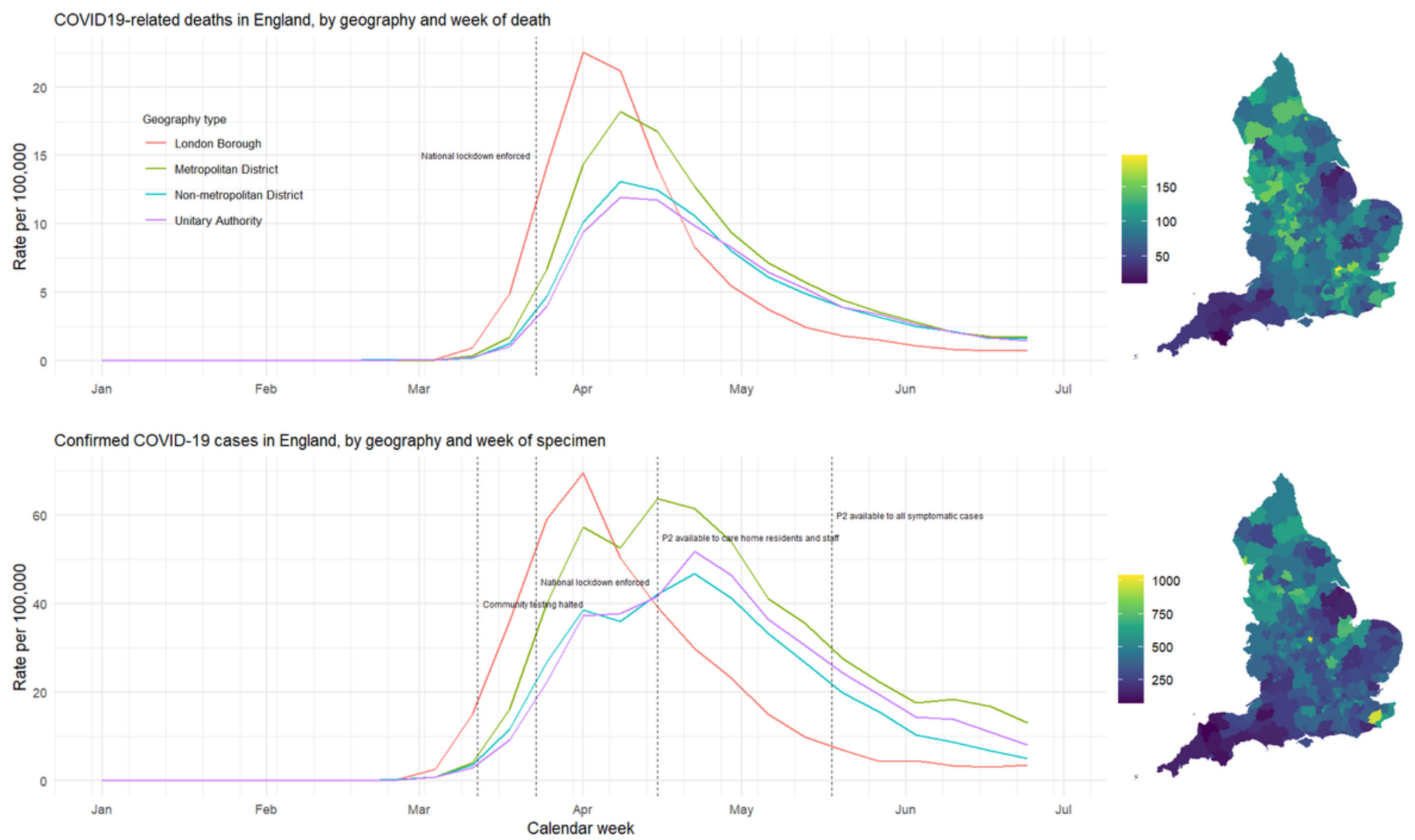

Figure 1

Rates of COVID-19-related deaths and confirmed cases in England, by geography and week of death, and by lower-tier local authority (LTLA). (A, B): Weekly rates per 100,000 population of COVID-19-related deaths and confirmed cases, respectively, by geography type. Trajectories of reported deaths follow a smooth epidemic curve while the peak in case counts appears to be truncated across geographies outside of the early-affected London region, potentially as a result of national lockdown measures but also of testing constraints. Dashed vertical lines mark dates of significant policy changes with respect to confirmatory testing of suspect cases. (C, D): The same data instead presented as total rates per 100,000 per LTLA, across the entire first wave (2020-01-01 to 2020-06-30). Time periods are set according to the date of specimen and date of death, respectively. 

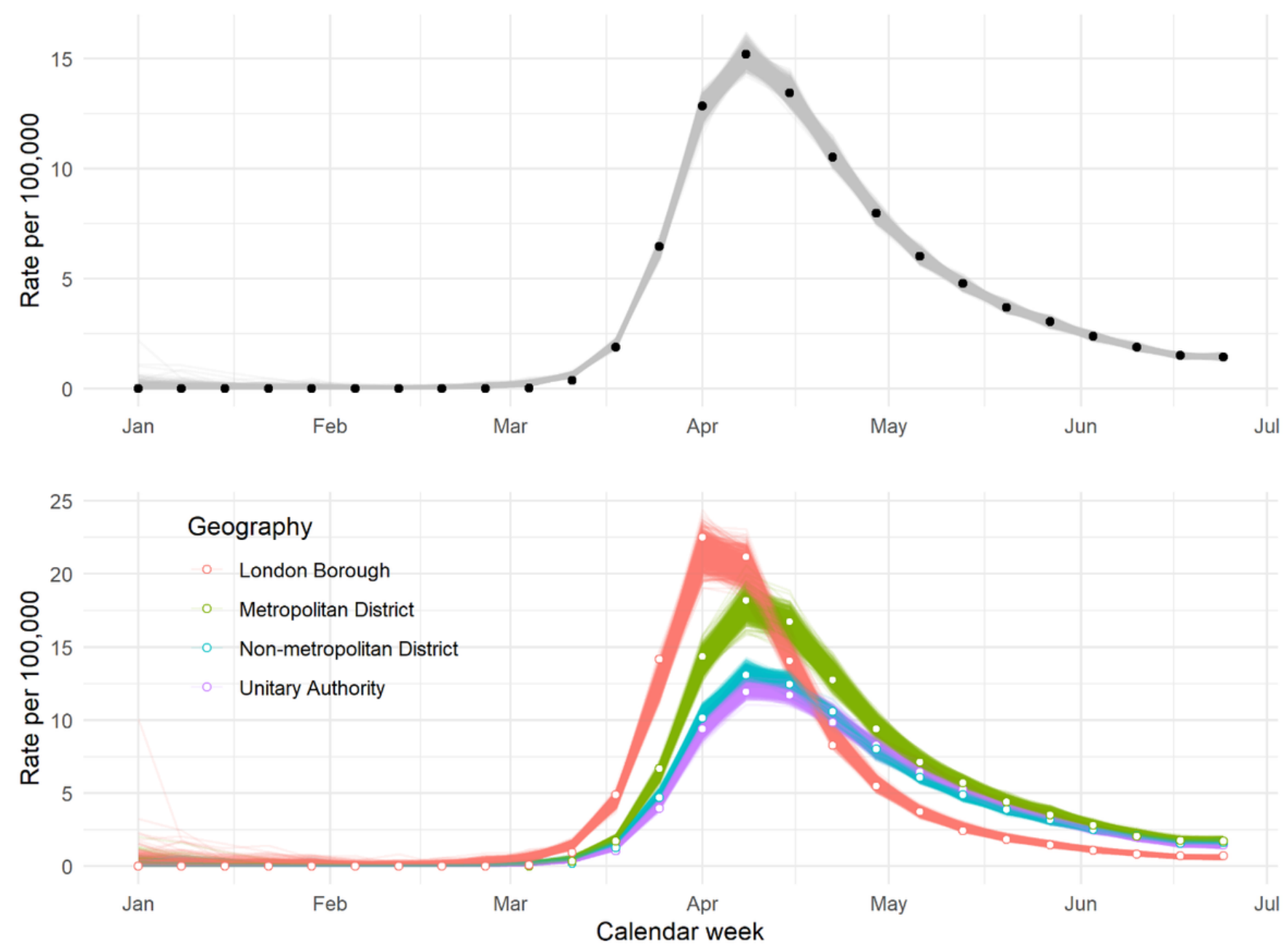

\section{Figure 2}

Final model fit (1,000 posterior samples) over time, as a national total and by geography type. The final model describes observed weekly COVID-19-related deaths per LTLA in terms of the size, age, ethnicity and deprivation level of the population, temporal trend and spatial correlation between neighbouring LTLAs. Observed rates of covid-related death per 100,000 population are shown in black (top) and white (bottom). Each grey/coloured line represents one sampled trajectory from the fitted model, and variation between these reflects uncertainty in the fit.
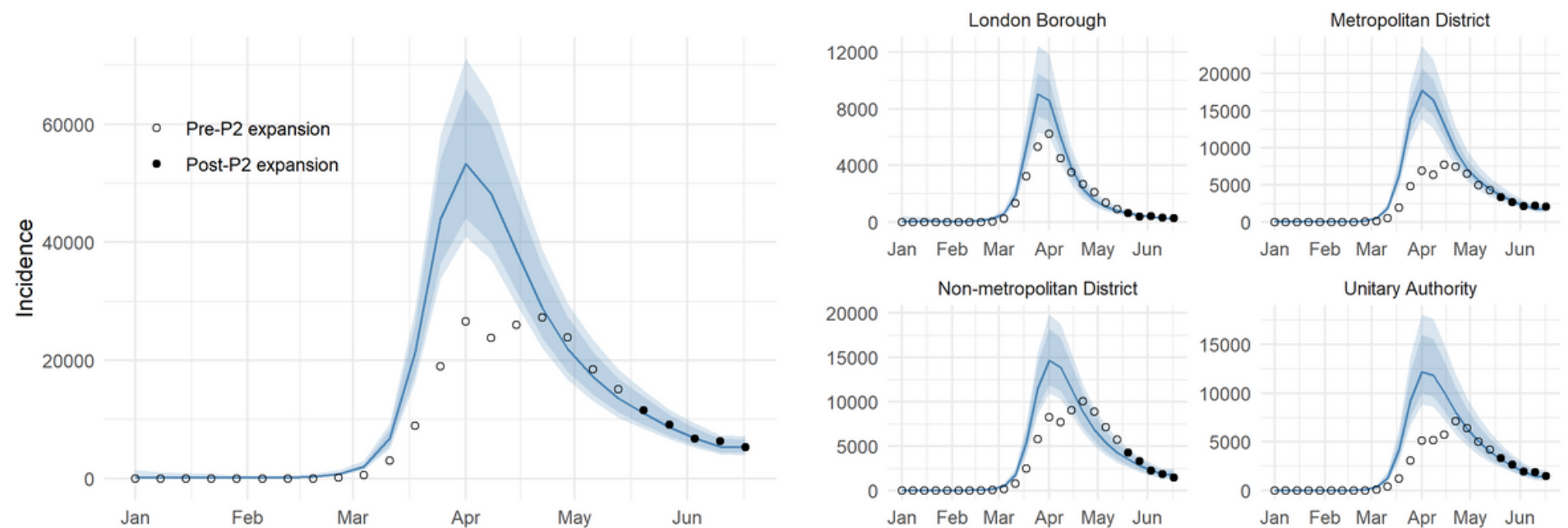

Figure 3 

geography type (right). Intervals shown are 50-98\% quantiles of the predicted distribution. Observed totals of confirmed cases per week are indicated by black points - unfilled prior to $P 2$-expansion and filled post-P2 expansion. Predicted-P1+P2 cases suggest the potential shape and magnitude of the first wave peak if community symptomatic testing (pillar 2) - in addition to hospital-based testing (pillar 1) - had been available from the beginning of the epidemic.

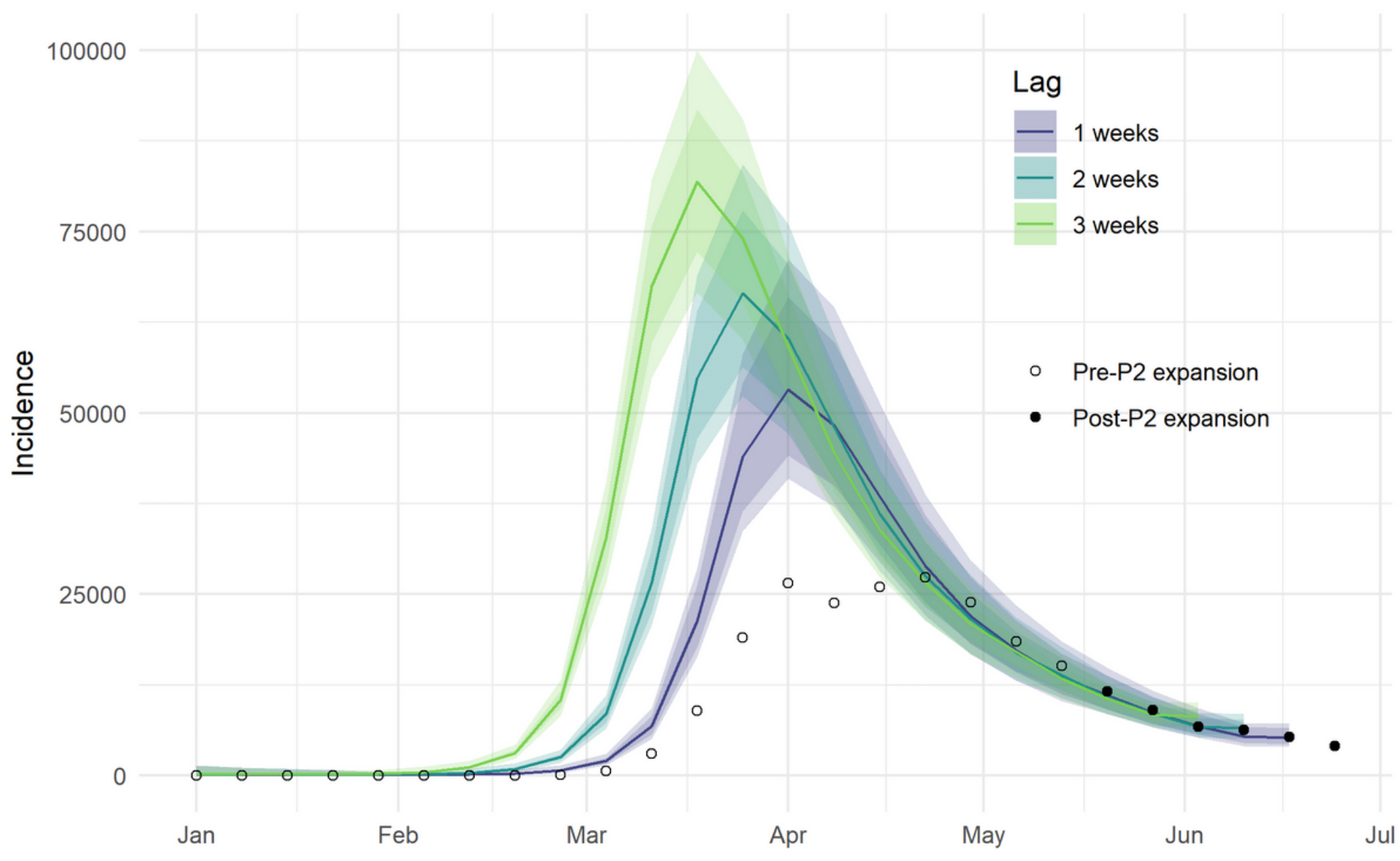

Figure 4

Comparison of predicted-P1+P2 cases assuming one, two- and three-week lags between date of swabbing and date of death. Shaded intervals represent 50 $98 \%$ quantiles of the predicted distribution. 
A
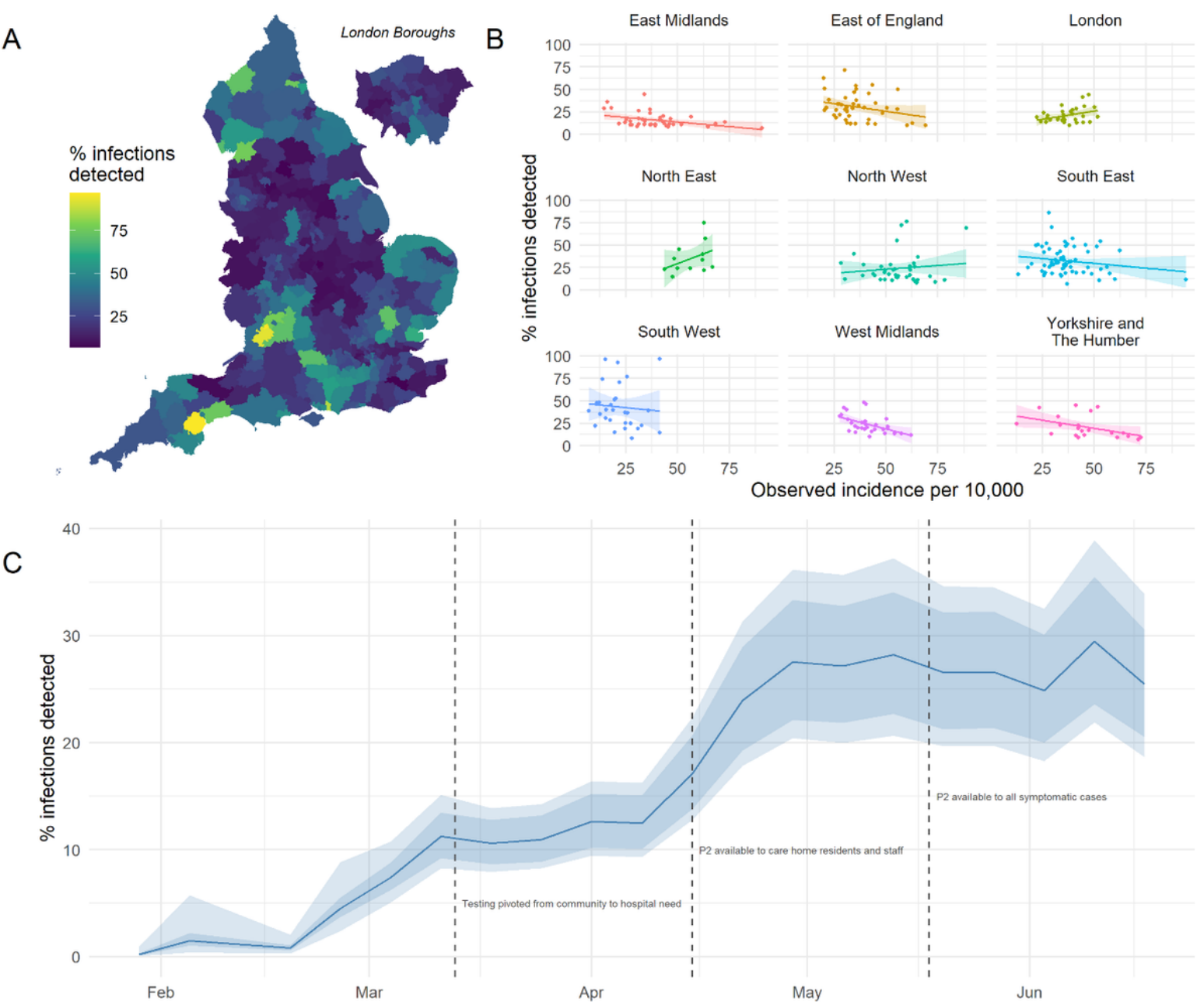

Figure 5

Estimated weekly incidence of infections in England (grey), inferred from predicted post-P2-detectable infections (blue) and an assumed detection rate of 25\% under expanded surveillance. Rate of detection is estimated by comparison of incidence estimates from the ONS infection survey (shown in red) and observed case counts (shown in black) between weeks starting 18th May to 15th June. This rate is then applied to predicted-P1+2 cases to obtain the estimated trajectory of total infections.

\section{Supplementary Files}

This is a list of supplementary files associated with this preprint. Click to download.

- SupplementaryMaterialsE.pdf

- SupplementaryMaterials.docx 